\title{
Genome-wide analysis of microRNA to evaluate prognostic markers in isolated cancer glands and surrounding stroma in high-grade serous ovarian carcinoma
}

\author{
CHIE SATO $^{1,2^{*}}$, MITSUMASA OSAKABE $^{1 *}$, TAKAYUKI NAGASAWA ${ }^{2}$, HIROMU SUZUKI $^{3}$, \\ HIROAKI ITAMOCHI $^{2}$, TSUKASA BABA ${ }^{2}$ and TAMOTSU SUGAI ${ }^{*}$ \\ Departments of ${ }^{1}$ Molecular Diagnostic Pathology and ${ }^{2}$ Obstetrics and Gynecology, School of Medicine, \\ Iwate Medical University, Yahaba, Iwate 028-3695; ${ }^{3}$ Department of Molecular Biology, \\ Sapporo Medical University School of Medicine, Sapporo, Hokkaido 060-8556, Japan
}

Received February 23, 2020; Accepted September 3, 2020

DOI: $10.3892 / \mathrm{ol} .2020 .12198$

\begin{abstract}
The molecular mechanisms responsible for the progression of ovarian cancer remain incompletely understood. By targeting multiple cancer-related genes, microRNAs (miRNAs) have been identified as key regulators of cancer development and progression. In addition, the microenvironment, which constitutes cancer glands and the surrounding stromal tissue at the invasive front, has an important role in cancer progression. Using array-based analysis of 14 cases (cohort 1), the aim of the present study was to evaluate global miRNA expression in cancerous glands and surrounding stromal tissues (isolated using a crypt isolation method), in order to identify potential prognostic markers of high-grade serous carcinoma (HGSC). Reverse transcription-quantitative PCR was also used to verify the results in cohort 1 (14 cases) and in 16 additional HGSC cases (cohort 2; verification cohort). Firstly, miRNA expression levels were compared between HGSC and normal samples among both the isolated cancer gland and stromal tissue samples. Secondly, miRNA expression was compared between HGSC cases with recurrence and those without recurrence among the isolated cancer gland and stromal tissue samples. The results revealed six and seven miRNAs identified in both of the aforementioned comparisons in isolated cancer glands and surrounding stromal tissue, respectively. Furthermore, downregulation of miRNA-214-3p in isolated cancer glands and downregulation of miRNA-320c in the corresponding stromal tissue were associated with a decrease
\end{abstract}

Correspondence to: Dr Tamotsu Sugai, Department of Molecular Diagnostic Pathology, School of Medicine, Iwate Medical University, 2-1-1 Idaidori, Yahaba, Iwate 028-3695, Japan

E-mail: tsugai@iwate-med.ac.jp

${ }^{*}$ Contributed equally

Key words: crypt isolation, genome-wide analysis, high-grade serous carcinoma, microRNA, stromal cell in disease-free survival (without recurrence) in cohort 2. These findings indicated that specific miRNAs expressed in cancer cells and surrounding stromal cells of HGSC may be potential biomarkers predicting patient prognosis.

\section{Introduction}

Ovarian cancer is the deadliest gynecological cancer in developed countries and, in Japan, the fifth leading cause of cancer death in women (1), with 4,745 deaths reported in 2017 (Cancer Registry and Statistics. Cancer Information Service, National Cancer Center, Japan. https://ganjoho.jp/reg_stat/statistics/dl/index.html). Ovarian cancer is typically diagnosed at an advanced stage, after peritoneal dissemination and massive ascites have developed (2,3). Furthermore, no improvement in the 5-year survival rate of ovarian cancer patients is evident, despite aggressive treatments involving surgery combined with platinum- and taxane-based chemotherapy (2-5). The most reliable prognostic factor predicting patient outcome is the TNM stage. Whereas stage I ovarian cancer has a good prognosis, the prognosis of stage IV is thought to be poor. According to this finding, to identify prognostic factors predicting the outcomes of patients with ovarian cancer, the objective of the study should be limited to patients with intermediate stages (i.e. stages II and III). Although pelvic examination, transvaginal ultrasonography, and serum CA125 measurement are used as routine diagnostic modalities, they fail to predict the prognosis of ovarian cancer patients (6). Therefore, new approaches to identify ovarian cancer markers are urgently needed $(7,8)$. At the molecular level, several genes and pathways have been identified that may be closely associated with the pathogenesis of ovarian cancer $(9,10)$; however, effective molecular markers predicting patient prognosis have not been established $(7,8)$. As potential candidates, microRNAs (miRNAs) regulate cancer-related gene expression and have been implicated in the etiology of ovarian cancer $(9,10)$. Recent studies have shown that dysregulation of miRNA is closely associated with patient prognosis in various cancers including ovarian cancer, melanoma and oral cancer (9-16). In addition, expression of miRNA might be useful as a diagnostic marker for cancer (9-16). 
A recent study showed that cancer-associated fibroblasts (CAFs), which are involved in the dynamic interaction between cancer cells and the unique tumor microenvironment, influence tumor progression as well as other genetic and epigenetic events that markedly affect disease outcome and treatment response 1 (17-20). Based on this theory, both cancer cells and the surrounding stromal cells play major roles in cancer progression (17-20).

We used high-throughput genome-wide miRNA analysis to evaluate the expression of specific miRNAs in cancer glands and stromal cells, obtained using a crypt isolation method that separates cancer glands from stromal cells, in ovarian high-grade serous carcinoma (HGSC), a common histological and lethal ovarian cancer variant.

\section{Materials and methods}

Patients. Thirty patients with HGSC of the ovary diagnosed at Iwate Medical University were enrolled. These patients were divided into cohort 1 (14 cases), whose samples were subjected to comprehensive microarray analyses, and cohort 2 (16 cases in addition to the 14 cases in cohort 1), whose samples were used to verify the results from cohort 1 by RT-qPCR. The HGSC samples were obtained from primary surgery in patients who had not received chemotherapy. Two expert pathologists determined the histological diagnoses, according to the General Rules for Ovarian Cancer of the Japan Gynecological Cancer Group (21), using hematoxylin and eosin staining to identify representative tumor areas, from which cores for microarray analysis were obtained. The TNM classification of the Union for International Cancer Control was used for disease staging (22). Cases of low-grade serous carcinoma were excluded from this study. The clinicopathological variables examined, summarized in Table I, included age, tumor size, FIGO stage, disease-free survival, and overall survival. All patients provided written informed consent, and the study was approved by the Iwate Medical University Institutional Review Board (approval no. MH2018-528).

Gland isolation. Crypts were isolated from tumor and normal tissues, as described previously, to obtain pure tumor glands separately from the surrounding stromal tissues (23-25). Tumor glands were isolated from the solid tumor region involved in the invasion front, and this involvement was confirmed using tissue sections prepared for the pathological diagnosis. Gland cells were obtained from the tumor tissues after careful separation of the stromal cells (i.e. CAFs) adjacent to the glands, performed under a dissecting microscope. Cells from normal fallopian tube tissue and normal fibroblasts within the Fallopian tubes were also obtained as controls. Paraffin-embedded tissue sections of the isolated samples were routinely processed to confirm the histology. Immunostaining using antibodies against smooth muscle actin (clone 1A4; Dako) and desmin (clone D33; Dako) was performed in the stromal cells to confirm the exclusive presence of fibroblasts, determined according to negative staining of smooth muscle actin and positive staining of desmin. However, we could not exclude the possibility of stromal cell contamination with other non-epithelial cells, such as inflammatory and vessel cells. Representative images are shown in Fig. 1.
RNA extraction. miRNAs in isolated tumor glands and the corresponding stromal cells were extracted using the mirVana $^{\mathrm{TM}}$ miRNA Isolation kit (Thermo Fisher Scientific, Inc.) following the manufacturer's instructions. The quantity and quality of the obtained RNA were evaluated using the DU730 spectrophotometer (Beckman Coulter) and the integrity by gel electrophoresis.

miRNA microarray analysis. For microarray analysis, $200 \mathrm{ng}$ RNA was polyadenylated and labelled using a FlashTag ${ }^{\mathrm{TM}}$ Biotin HSR RNA Labelling kit and then treated with DNA ligase. The labeled RNA was hybridized to GeneChip miRNA 4.0 microarrays (Thermo Fisher Scientific) at $48^{\circ} \mathrm{C}$ for $16 \mathrm{~h}$, followed by washing and staining using a streptavidin-PE solution. The stained arrays were scanned using a GeneChip ${ }^{\mathrm{TM}}$ Scanner 3000 7G System (Thermo Fisher Scientific). The Affymetrix miRNA 4.0 microarray contains 6,631 probes on the array, including 2,570 mature miRNA probes. Detailed methods have been described previously (26).

Verification of miRNA expression by RT-qPCR in cohort 2 samples. RT-qPCR was used to confirm miRNA expression levels in HGSC (isolated cancer glands and surrounding stromal cells) and normal tissue samples using the Applied Biosystem (ABI) Detection System (Step One Plus) (27). First, cDNA was reverse-transcribed from $10 \mathrm{ng}$ total RNA using the TaqMan MicroRNA Reverse Transcription Kit (cat. no. 4366596; ABI). Then, PCR was performed using TaqMan Universal Master Mix II, no UNG (cat. no.: 4440040), and the following TaqMan assays: Hsa-miR-188-5p (assay ID: 002320), hsa-miR-214-3p (assay ID: 002306), hsa-miR-505-5p (assay ID: 002087), hsa-miR-4455 (assay ID: 463355_mat), hsa-miR-6753-3p (assay ID: 466443_mat) and hsa-miR-6877-3p (assay ID: 467048_mat). In addition, the expression levels of hsa-miR-101-5p (assay ID: 002143), hsa-miR-320c (assay ID: 241053_mat), hsa-miR-320d (assay ID: 241066_mat), hsa-miR-320e (assay ID: 243005_mat), hsa-miR-378f (assay ID: 462794_mat), hsa-miR-455-3p (assay ID: 002244), hsa-miR-4429 (assay ID: 464083_mat) in the isolated stromal cells were examined by the same method. All TaqMan miRNA assays were obtained from ABI. The primer sequences are provided in Table SI. The $2^{-\Delta \Delta \mathrm{Cq}}$ method was used to determine the relative expression levels, with hsa-let-7a as an internal control that was determined based on pre-analytical experiments in which expression levels of RNU6B, let-7a and miRNA-21 were examined in all samples including isolated cancer glands and isolated stromal tissue samples. The expression levels of let-7a were constant and stable in both isolated cancer gland and isolated stromal tissue samples.

Statistical analysis. Differences in miRNA expression levels were analyzed using the TAC4.0 (Thermo Fisher Scientific Inc.) and JMP pro 13.0 software package for Windows (SAS Institute Inc.).

Expression levels of miRNAs were analyzed using Mann-Whitney $U$ test. We determined the cutoff expression levels using receiver operating characteristic (ROC) analysis. We calculated disease-free survival (without recurrence including metachronous metastasis) from the date of surgery to the development of recurrence (including metachronous 
Table I. Clinicopathological findings of the ovarian high-grade serous carcinoma we examined.

\begin{tabular}{|c|c|c|}
\hline Variable & Cohort $1^{\mathrm{a}}$ & Cohort $2^{b}$ \\
\hline Total & 14 & 30 \\
\hline $\begin{array}{l}\text { Median age (range), } \\
\text { years }\end{array}$ & $46.5(31-79)$ & $56(31-79)$ \\
\hline $\begin{array}{l}\text { Tumor size, median } \\
\text { (range), mm }\end{array}$ & $95(28-176)$ & $89.5(20-200)$ \\
\hline \multicolumn{3}{|l|}{ FIGO stage $(\%)$} \\
\hline II & $2(14.3)$ & $8(26.7)$ \\
\hline III & $12(85.7)$ & $22(73.3)$ \\
\hline \multicolumn{3}{|l|}{ Recurrence (\%) } \\
\hline Present & $7(50.0)$ & $16(53.3)$ \\
\hline Absent & $7(50.0)$ & $14(46.7)$ \\
\hline \multicolumn{3}{|l|}{ Survival (\%) } \\
\hline Dead & $2(14.3)$ & $6(20.0)$ \\
\hline Alive & $12(85.7)$ & $24(80.0)$ \\
\hline $\begin{array}{l}\text { Disease free survival, } \\
\text { median (range), days }\end{array}$ & $637(381-1,098)$ & $592(25-2,166)$ \\
\hline $\begin{array}{l}\text { Overall survival, } \\
\text { median (range), days }\end{array}$ & $1,035(400-3,343)$ & $1,084(259-3,343)$ \\
\hline
\end{tabular}

${ }^{a}$ Comprehensive analysis using microRNA array; ${ }^{b}$ validation test.

metastasis) or the last follow-up, and overall survival from the date of surgery to death or the last follow-up using Kaplan-Meier analysis. A log rank test was conducted after Kaplan-Meier analysis to determine significance. Potential factors associated with survival were identified by univariate and multivariate analyses using Cox proportional hazards regression models conducted using JMP 13.0 software. $\mathrm{P}<0.01$ and $\mathrm{P}<0.05$ were considered significant in array analysis and non-array analysis, respectively.

\section{Results}

Association of clinicopathological findings between cohort 1 and 2. There was no significant difference in the clinicopathological variables between cohorts 1 and 2 .

miRNA expression profiling in ovarian HGSC. To identify potential miRNA biomarkers of ovarian HGSC, we performed global miRNA expression profiling in the 14 HGSCs and normal tissues samples, as well as in the corresponding surrounding stromal and normal fibrous tissues. Using the criteria of a fold change in expression $<-2$ or $>2$ and $\mathrm{P}<0.01$, we performed two comparisons among the isolated cancer gland samples. First, we compared expression levels between isolated cancer glands and normal crypts and identified 330 differentially expressed miRNAs (115 downregulated and 215 upregulated). Second, we compared miRNA expression in isolated cancer glands between HGSC cases with recurrence (including metachronous metastasis) and those without recurrence (including metachronous metastasis) and identified
26 differentially expressed miRNAs (18 downregulated and 8 upregulated). Of these differentially expressed miRNAs, six (hsa-miR-188-5p, hsa-miR-214-3p, hsa-miR-505-5p, hsa-miR-4455, hsa-miR-6753-3p, and hsa-miR-6877-3p) were identified as differentially expressed (two significantly downregulated and four significantly upregulated) in both comparisons in isolated cancer glands, based on a Venn diagram (Fig. 2A).

Using the same differential expression criteria (fold change $<-2$ or $>2$ and $\mathrm{P}<0.01$ ), we performed two comparisons in the isolated stromal cell samples, similar to those made in the isolated cancer glands. We identified 324 differentially expressed miRNAs (102 significantly downregulated and 222 significantly upregulated) between cancer and normal stromal cells and 21 differentially expressed miRNAs (13 significantly downregulated and 8 significantly upregulated) in cancer stromal cells between HGSC cases with recurrence (including metachronous metastasis) and those without recurrence. Of these differentially expressed miRNAs, seven (hsa-miR-101-5p, hsa-miR-320c, hsa-miR-320d, hsa-miR-320e, hsa-miR-378f, hsa-miR-455-3p and hsa-miR-4429) were identified as differentially expressed (six significantly downregulated and one significantly upregulated) in both sets of comparisons in stromal cells, based on a Venn diagram (Fig. 2B).

Fold-changes in miRNA expression for each comparison (e.g., recurrence vs. no recurrence; crypt vs. stroma) are presented in Table SII.

Comparison of candidate miRNA expression between cancer gland and stromal tissue samples in cohorts 1 and 2. Among the six differentially expressed miRNAs identified in isolated cancer glands from patients in cohort 1 , statistically significant differences in the expression level were seen for four miRNAs (hsa-miR-188-5p, hsa-miR-214-3p, hsa-miR-4455 and hsa-miR-6877-3p) in comparisons of HGSCs with recurrence (including metachronous metastasis) and without recurrence. Meanwhile, for patients in cohort 2, two miRNAs (hsa-miR-188-5p and hsa-miR-214-3p) had statistically different expression levels between HGSCs with recurrence (including metachronous metastasis) and without recurrence (including metachronous metastasis) (Fig. 3Aa-f and Ba-f). Of the seven differentially expressed miRNAs identified in isolated stromal cells from cohort 1 , differences were seen in the expression level of five miRNAs including hsa-miR-101-5p, hsa-miR-320c, hsa-miR-320d, hsa-miR-320e, hsa-miR-455-3p and hsa-miR-4429 between HGSCs with recurrence (including metachronous metastasis) and those without recurrence (cohort 1). In cohort 2, four miRNAs (hsa-miR-320c, hsa-miR-320d, hsa-miR-378f, and hsa-miR-4429) had statistically different expression levels between HGSCs with recurrence (including metachronous metastasis) and those without recurrence (Fig. 3Ag-m and Bg-m).

Ability of miRNA expression to predict patient survival. We determined the cutoff expression levels of miRNA-214-3p and miRNA-320c that are potentially predictive of the development of recurrence (metachronous metastasis) in HGSC, using receiver operating characteristic (ROC) analysis (Fig. S1). In the present study, we did not perform absolute quantification 

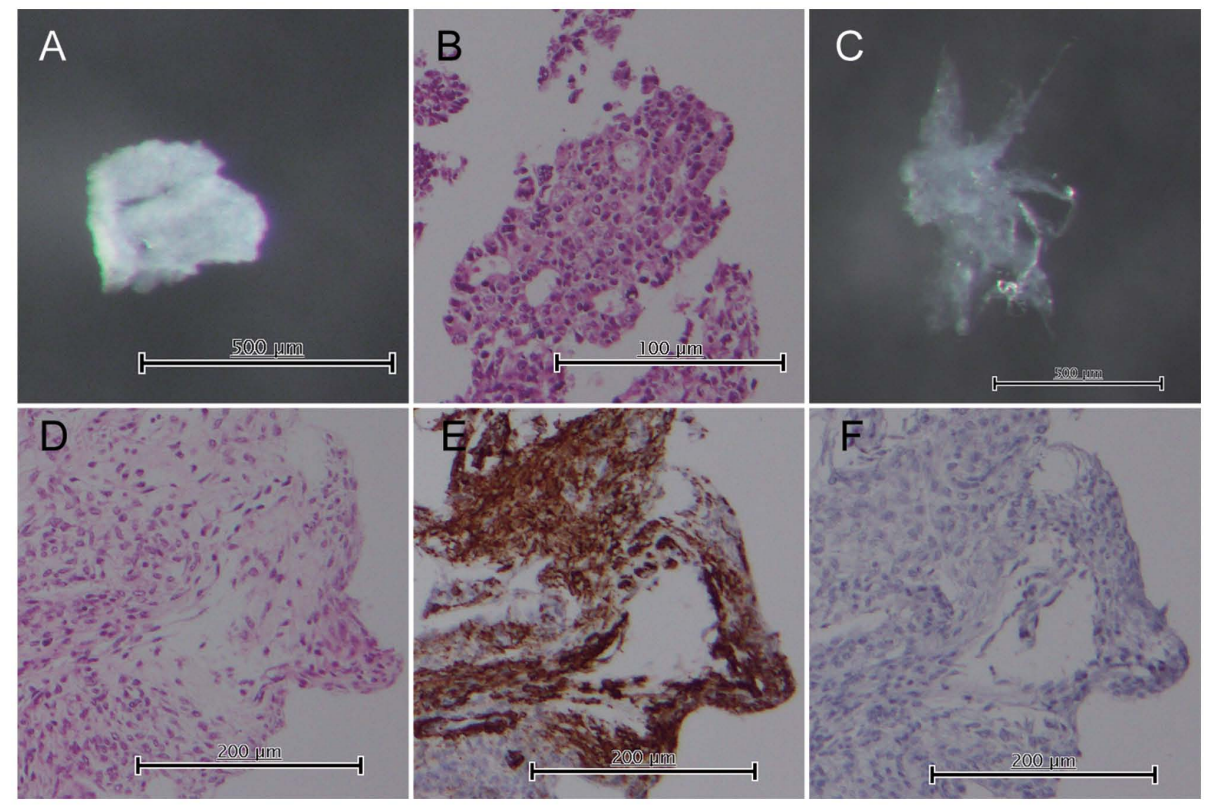

Figure 1. Representative figure of isolated cancer glands and stromal cells. (A) Ovarian cancer glands observed under a dissecting microscope (tumor crypt: Large glands or sheets of epithelium). (B) Isolated carcinoma gland illustrated by hematoxylin and eosin staining of tissue sections. This histological section shows a high-grade serous carcinoma. (C) Ovarian cancer stromal cells observed under a dissecting microscope (large sheet resembling fibrous tissue with an irregular contour). (D) Histology of cancer-associated fibroblasts according to hematoxylin and eosin staining of tissue sections. (E) Positive immunostaining of smooth muscle actin. (F) Negative immunostaining of desmin. Accordingly, this fibrous tissue was considered to contain activated fibroblasts that are regarded as cancer-associated fibroblasts.
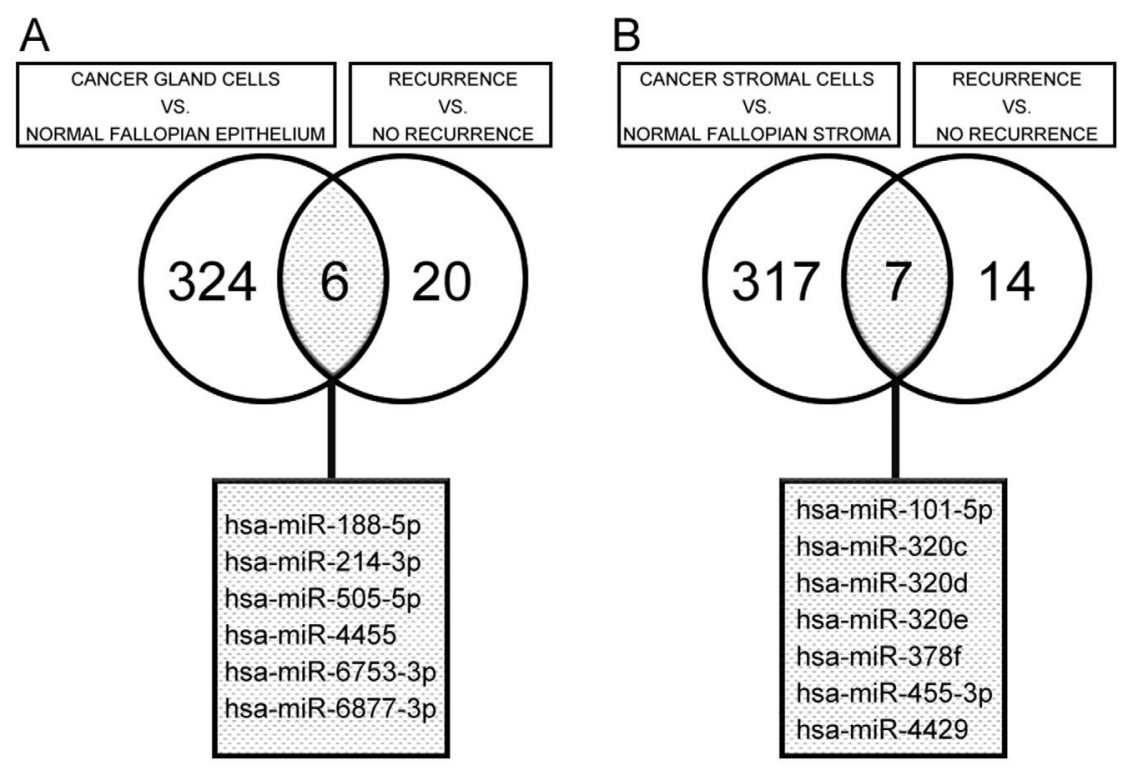

Figure 2. Venn diagrams of differentially expressed miRNAs determined by miRNA microarray analysis. (A) Cancer gland cells. (B) Cancer stromal cells. miRNA/miR, microRNA.

in order to determine standard value for prediction of patient prognosis with HGSC. At each expression level, the sensitivity and specificity for the outcome under study (recurrence including metachronous metastasis) were plotted to generate a ROC curve. If a ROC curve was generated from pairs of weighted mean sensitivities and mean specificities, then the weighted mean sensitivities and specificities for each miRNA expression level were plotted to generate ROC curves, and the area under the curve was used to determine the ability of the miRNA to discriminate between the presence and absence of recurrence (including metachronous metastasis). As a result, miRNA-214-3p and miRNA-320c expression levels were identified as the best predictors of recurrence (including metachronous metastasis) in HGSC among the miRNAs examined (area under the curve: 0.81696 for isolated cancer glands and 0.88393 for isolated stromal tissue). Consequently, less than 0.278271 for miRNA-214-3p expression level (Fig. S1 Ab) and less than 0.198724 for miRNA-320c expression level (Fig. S1Bb), respectively, determined by RT-qPCR were regarded as presence of downregulation. 
A a

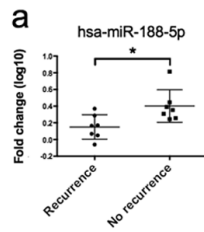

g

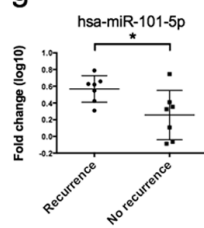

B a
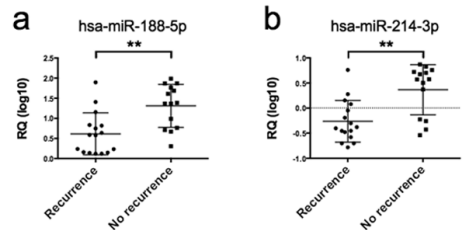

$\mathrm{h}$

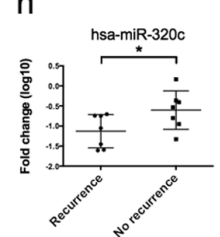

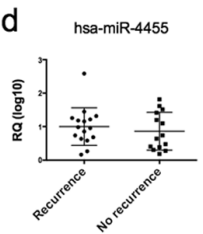
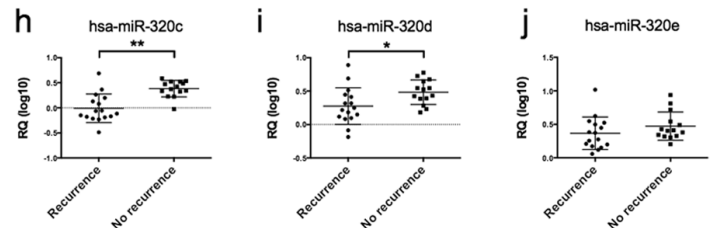
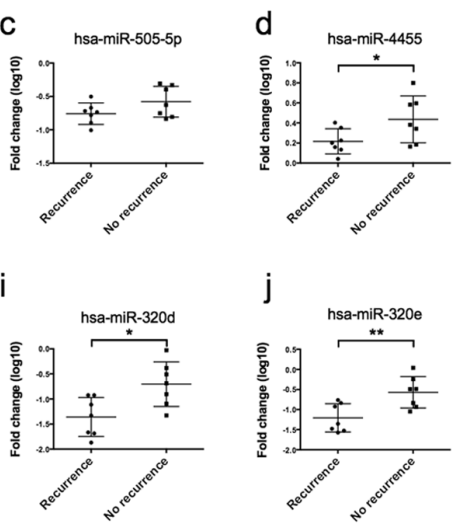

j
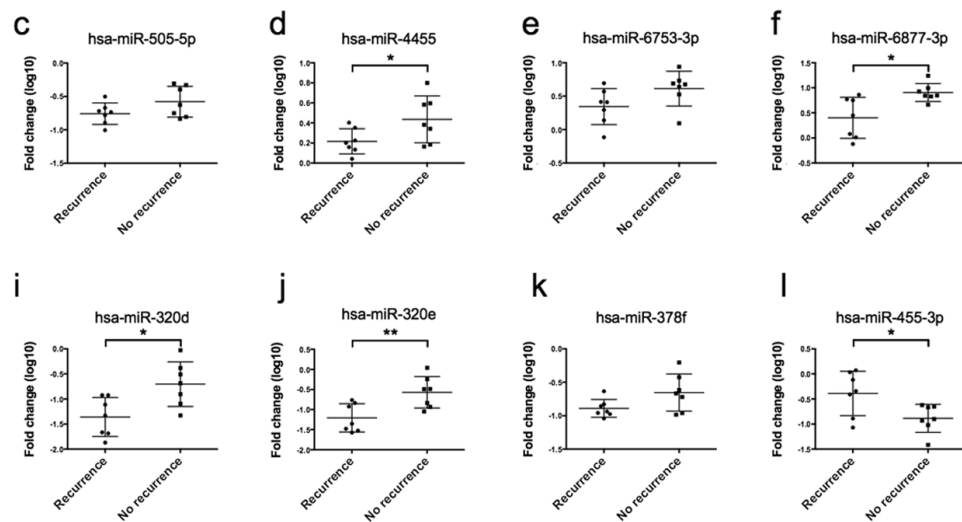

k

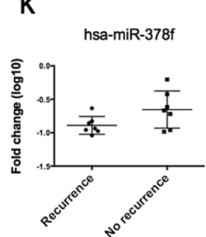

I

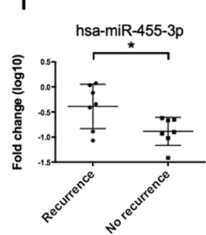

m

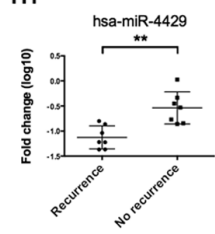

f hsa-miR-6877-3p

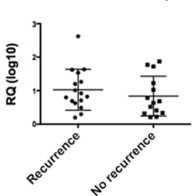

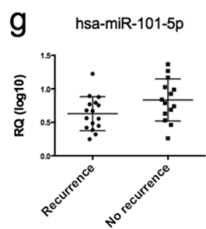
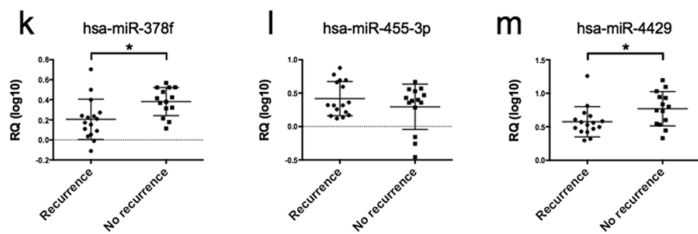

Figure 3. Expression levels of microRNAs in the high-grade serous carcinoma cases with recurrence and those without recurrence. (A) Cohort 1 (miRNA microarray analyses). (B) Cohort 2 (Verification tests). (a) hsa-miR-188-5p. (b) hsa-miR-214-3p. (c) hsa-miR-505-5p. (d) hsa-miR-4455. (e) hsa-miR-6753-3p. (f) hsa-miR-6877-3p (g) hsa-miR-101-5p. (h) hsa-miR-320c. (i) hsa-miR-320d. (j) hsa-miR-320e. (k) hsa-miR-378f. (l) hsa-miR-455-3p. (m) hsa-miR-4429. (a-f) Isolated cancer gland samples; (g-m) isolated stromal samples. ${ }^{*} \mathrm{P}<0.05,{ }^{* *} \mathrm{P}<0.01$; statistics, Mann-Whitney U test. RQ, relative quantification; miRNA/miR, microRNA.

Association of clinicopathological findings with candidate miRNAs identified in cancer gland and stromal tissue samples. We examined associations of clinicopathological findings including sex, age, tumor size and histological grade with expression of miRNA-214-3p and miRNA-320c in samples from isolated cancer glands and stromal tissues, respectively. In univariate analysis of clinicopathological findings, we observed no significant difference in the expression of miRNAs including miRNA-214-3p and miRNA-320c.

Associations between patient survival and the candidate miRNAs identified in the cancer gland and stromal tissue samples. Of the 30 HGSC cases, 14 (46.7\%) had no recurrence (including metachronous metastasis). Disease-free survival (without recurrence) was compared according to the expression of each miRNA differentially expressed in the isolated cancer gland samples using Kaplan-Meier analysis (Fig. 4A). The presence of recurrence including metachronous metastasis was associated with downregulation of hsa-miRNA-214-3p. Cox proportional hazards models were used to determine any independent associations of disease-free survival with the clinicopathological findings and hsa-miRNA-214-3p expression. We initially performed univariate analyses to determine the associations of the following variables with the presence of recurrence including metachronous metastasis: Age, tumor size, FIGO stage, and the six differentially expressed miRNAs identified in the isolated cancer glands. The results indicated that FIGO stage and expression of hsa-miRNA-188-5p and hsa-miRNA-214-3p were associated with the presence of recurrence including metachronous metastasis. The FIGO stage and hsa-miRNA-214-3p expression retained significance in the multivariate analysis. The summarized results are shown in Table II.

Using the same process, the association of the expression of specific miRNAs with disease-free survival was examined in isolated stromal tissue. According to Kaplan-Meier analysis, downregulation of hsa-miRNA-320c was associated with the patients who had developed recurrence including metachronous metastasis (Fig. 4B). Then, univariate analyses were conducted to determine whether age, tumor size, FIGO stage, and the seven candidate miRNAs identified in isolated stromal tissue were independent predictors of disease-free survival in HGSC patients. According to the results, the FIGO stage and hsa-miRNA-320c expression were associated with an increased rate of recurrence including metachronous metastasis, and both factors remained significant in the multivariate analysis. These results are summarized in Table III.

\section{Discussion}

The cancer microenvironment, comprising cancer cells and adjacent stromal cells, was recently reported to have a key role in human cancer pathogenesis $(17,18)$. However, despite advances in molecular cancer science, for ovarian cancer the 
A a
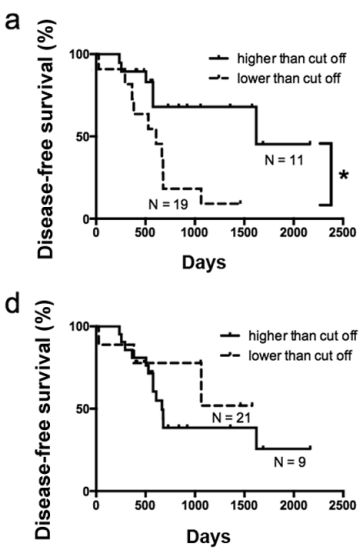

Days
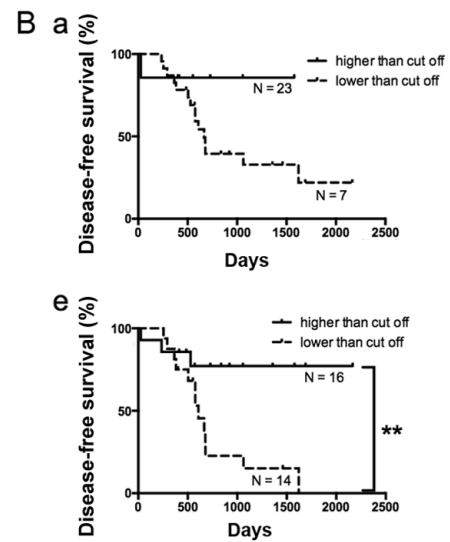
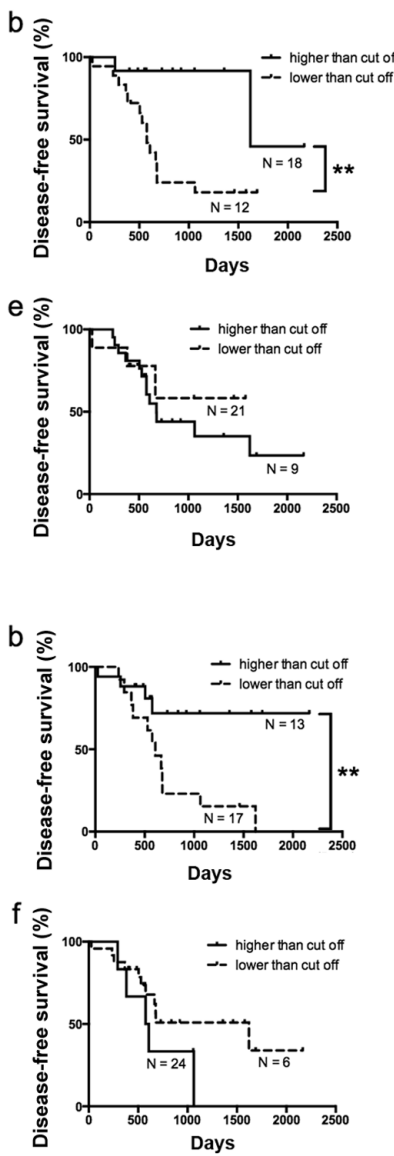
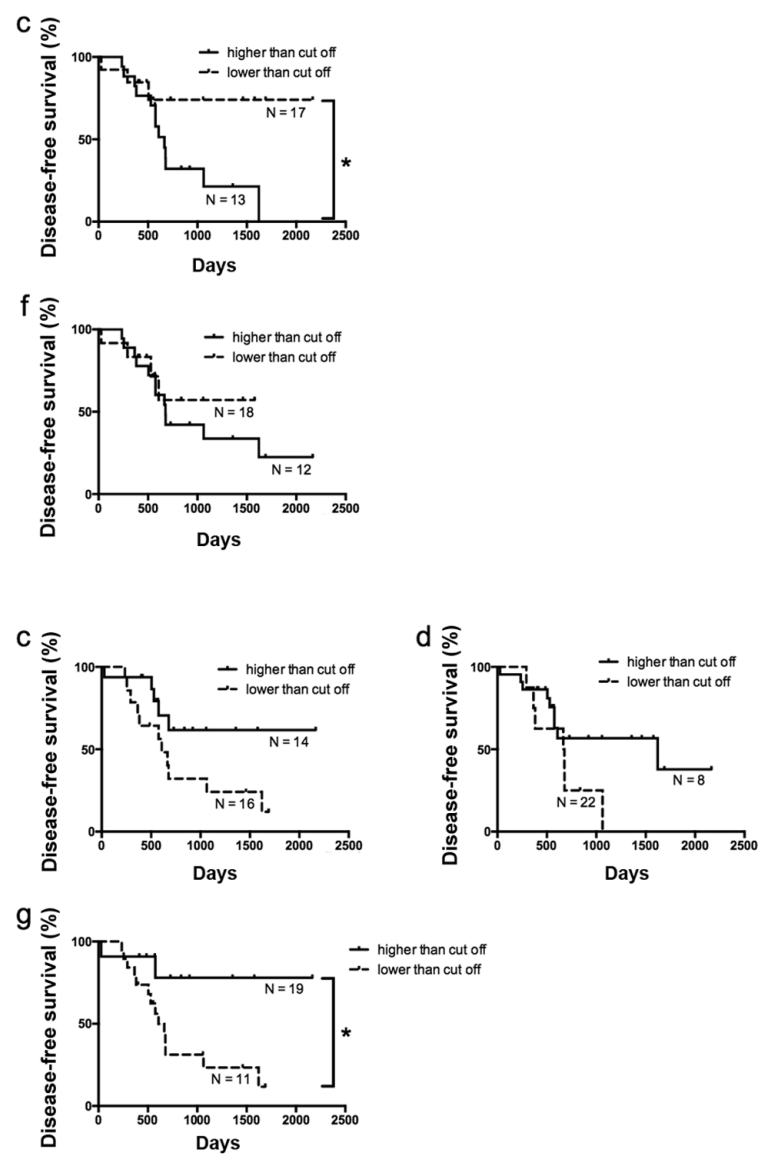

Figure 4. Kaplan-Meier analyses. (A) DFS according to distinct cutoff values in cancer gland cells. (a) hsa-miR-188-5p. (b) hsa-miR-214-3p. (c) hsa-miR-505-5p. (d) hsa-miR-4455. (e) hsa-miR-6753-3p. (f) hsa-miR-6877-3p.(B) DFS according to distinct cutoff values in cancer stromal cells. (g) hsa-miR-101-5p. (h) hsa-miR-320c. (i) hsa-miR-320d. (j) hsa-miR-320e. (k) hsa-miR-378f. (1) hsa-miR-455-3p. (m) hsa-miR-4429. ${ }^{*} \mathrm{P}<0.05$, ${ }^{* *} \mathrm{P}<0.01$. DFS, disease-free survival; miR, microRNA.

detailed molecular mechanisms associated with the cancer microenvironment remain unclear in part because ovarian cancer is a heterogeneous disease that is strongly influenced by genetic and epigenetic alterations $(28,29)$. Moreover, the tumor environment in ovarian cancer involves complex interconnected signaling networks $(17,18)$. CAFs interact with several immune cells, including tumor-associated macrophages, $\mathrm{T}$ cells, natural killer cells, and cytokines, to promote the growth and metastasis of ovarian cancer cells (30). Therefore, understanding the pathogenesis and unique tumor microenvironment, which may determine malignancy, of ovarian cancer is crucial for developing more sensitive tools predicting patient prognosis, in turn influencing the choice of effective treatment options $(11,12)$. We attempted to identify specific miRNAs potentially predicting the prognosis of patients with HGSC, a common variant of ovarian cancer, based on their expression levels in isolated cancer glands and surrounding stromal cells.

Here, we used a crypt isolation method to isolate both pure tumor glands as well as the surrounding stromal tissues. Although this method can accurately isolate pure tumor glands based on our extensive experience (23-25), it is possible that our isolated stromal cells were contaminated with cancer cells (23). To avoid such contamination, we confirmed that the histological sections of the isolated stromal tissues were devoid of cancer gland cells and comprised primarily CAFs. We believe that the cancer glands and stromal tissue were successfully separated in the present study.
Recently, significantly lower miRNA-214-3p expression was found in two esophageal squamous cancer cell lines relative to that in normal esophageal epithelial cells $(31,32)$. Another study demonstrated that reduced miRNA-214-3p expression inhibited chemoresistance in esophageal cancer cells by targeting survivin (an inhibitor of apoptotic proteins) and CUG-binding protein 1 (an RNA binding protein), which increases survivin expression (32). Maternal embryonic leucine zipper kinase (MELK), a member of the anti-apoptotic Bcl-2 family and essential for cancer growth, was identified as a target gene of miRNA-214-3p; MELK was also identified as a target gene of PRDI-BF1/Blimp-1, a transcriptional corepressor for specific subsets of DNA-binding transcription factors, suggesting a possible mechanism underlying the proliferation and resistance to apoptosis of hepatocellular carcinoma cells (32). This was supported by the association between MELK expression and gastric cancer progression detected in clinical samples (33). These findings suggest that decreased miRNA-214-3p expression affects the progression of specific cancers by targeting MELK (31,32). A recent study showed downregulated miRNA-214-3p expression and a negative correlation between miRNA-214-3p and fibroblast growth factor receptor 1 (FGFR1) expression in lung cancer patients (34). A regulatory mechanism involving miRNA-214-3p and FGFR1 was proposed, suggesting that miRNA-214-3p is an important therapeutic target in lung cancers with FGFRl gene amplification (34). Thus, miRNA-214-3p expression may play a crucial role in 
Table II. Univariate and multivariate analyses of clinicopathologic findings and miRNA expression as predictors of recurrence in cancer gland cells of ovarian high-grade serous carcinoma using Cox proportional hazards model.

\begin{tabular}{|c|c|c|c|c|c|c|}
\hline \multirow[b]{2}{*}{ Variables } & \multicolumn{3}{|c|}{ Univariate analysis } & \multicolumn{3}{|c|}{ Multivariate analysis } \\
\hline & HR & $95 \% \mathrm{CI}$ & P-value & HR & $95 \% \mathrm{CI}$ & $\mathrm{P}$-value \\
\hline Age (years) & 0.984 & $0.944-1.024$ & 0.4320 & & & \\
\hline Tumor size (mm) & 0.995 & $0.982-1.007$ & 0.4157 & & & \\
\hline \multicolumn{7}{|l|}{ FIGO stage } \\
\hline III vs. II & 8.455 & $1.680-153.834$ & 0.0056 & 14.745 & $2.185-321.081$ & 0.0029 \\
\hline \multicolumn{7}{|c|}{ miRNA expression in cancer gland cells } \\
\hline hsa-miR-188-5p & 0.376 & $0.123-0.965$ & 0.0416 & 4.462 & $0.572-35.610$ & 0.1510 \\
\hline hsa-miR-214-3p & 0.257 & $0.069-0.754$ & 0.0119 & 0.075 & $0.008-0.648$ & 0.0187 \\
\hline hsa-miR-4455 & 1.257 & $0.439-3.479$ & 0.6639 & & & \\
\hline hsa-miR-505-5p & 2.527 & $0.663-9.278$ & 0.1699 & & & \\
\hline hsa-miR-6753-3p & 1.182 & $0.432-3.060$ & 0.7368 & & & \\
\hline hsa-miR-6877-3p & 1.394 & $0.537-3.461$ & 0.4841 & & & \\
\hline
\end{tabular}

HR, hazard ratio; $95 \% \mathrm{CI}, 95 \%$ confidence interval; miRNA/miR, microRNA.

Table III. Univariate and multivariate analyses of clinicopathologic findings and miRNA expression as predictors of recurrence in cancer stroma of ovarian high-grade serous carcinoma using Cox proportional hazards model.

\begin{tabular}{|c|c|c|c|c|c|c|}
\hline \multirow[b]{2}{*}{ Variables } & \multicolumn{3}{|c|}{ Univariate analysis } & \multicolumn{3}{|c|}{ Multivariate analysis } \\
\hline & HR & $95 \% \mathrm{CI}$ & P-value & HR & $95 \% \mathrm{CI}$ & P-value \\
\hline Age (years) & 0.984 & $0.944-1.024$ & 0.4320 & & & \\
\hline Tumor size (mm) & 0.995 & $0.982-1.007$ & 0.4157 & & & \\
\hline \multicolumn{7}{|l|}{ FIGO stage } \\
\hline III vs. II & 8.455 & $1.680-153.834$ & 0.0056 & 5.926 & $1.074-110.573$ & 0.0438 \\
\hline \multicolumn{7}{|c|}{ miRNA expression in cancer stroma } \\
\hline hsa-miR-101-5p & 0.272 & $0.038-1.728$ & 0.1704 & & & \\
\hline hsa-miR-320c & 0.086 & $0.012-0.502$ & 0.0061 & 0.129 & $0.013-0.947$ & 0.0398 \\
\hline hsa-miR-320d & 0.145 & $0.018-1.234$ & 0.0767 & & & \\
\hline hsa-miR-320e & 0.212 & $0.011-2.506$ & 0.2327 & & & \\
\hline hsa-miR-378f & 0.062 & $0.003-1.013$ & 0.0511 & & & \\
\hline hsa-miR-455-3p & 3.198 & $0.632-23.207$ & 0.1707 & & & \\
\hline hsa-miR-4429 & 0.090 & $0.006-1.072$ & 0.0572 & & & \\
\hline
\end{tabular}

HR, hazard ratio; 95\% CI, 95\% confidence interval; miR/miRNA, microRNA.

human cancers by targeting specific proteins such as MELK and FGFR1 $(31,34)$. In the current study, downregulation of miRNA-214-3p was correlated with disease-free survival in isolated cancer glands obtained from primary HGSC specimens. As immunohistochemical expression of miRNA-214-3p target proteins, including MELK and FGFR1, was not examined in this study, further studies are needed to identify whether MELK and FGFR1 expression is related to downregulation of miRNA-214-3p in HGSC. Finally, this finding highlights a possible association of miRNA 214-3p expression with cancer-stroma interactions. The role of miR-214-3p in modulating expression of genes encoding proteins involved in extracellular matrix (ECM) degradation, tumor invasion and metastasis that is associated with the tumor microenvironment comprising heterogeneous cancer cells and surrounding interstitial cells has been the focus of several studies in the carcinogenesis field (34). For bladder cancer, down-regulation of miR-214-3p expression is associated with prognosis and modulation of MMP-9 and NGAL (Neutrophil gelatinase-associated lipocalin) gene expression and may reflect formation of the tumor microenvironment $(31,35,36)$. In addition, downregulated hsa-miR-214-3p expression could in turn alter the expression of genes involved in both epithelia mesenchymal transition (EMT) and NGAL/MMP-9 pathways, suggesting that downregulation 
of miR214-3p expression could facilitate EMT via activation of NGAL/MMP-9 pathways (35). The present study is the first to suggest that downregulation of miRNA-214-3p is correlated with disease-free survival in HGSC.

Despite reports that the miRNA-320 family is involved in several different human malignancies, its role in ovarian cancer is not fully understood $(37,38)$. In colorectal cancer, SOX4, FOXM1, and FOXQ1 were identified as novel targets of the miRNA-320 family (37), and Racl was found to be a direct target of miRNA-320a (39). Sun et al showed that miRNA-320a inhibits colorectal cancer cell growth by targeting the $\beta$-catenin signaling pathway (38). Interestingly, Zhang et al demonstrated that miRNA-320d was downregulated in stem cells derived from HT29 colon cancer cells expressing CD133 compared with those without CD133 expression, suggesting that miRNA-320 might be involved in stem cell differentiation (40). In another study, abnormal expression of miRNA-320 was examined in several human malignant tumors and found to be downregulated in malignant cholangiocarcinoma, in which this miRNA negatively regulated the expression of anti-apoptotic Mcl-1 and Bcl-2 (41). Furthermore, in prostate cancer, upregulation of miRNA-320 inhibited the Wnt/ $\beta$-catenin pathway and decreased CD44 expression, a marker of cancer stem cells, in tumor-initiating cells (42). However, little has been reported on the expression of the miRNA-320 family in CAFs, especially those in ovarian cancer. In the present study, downregulation of miRNA-320c in isolated stromal cells was correlated with disease-free survival in patients with HGSC. As a potential mechanism, decreased miRNA-320 expression in CAFs surrounding the tumor leads to upregulation of several miRNA-320 target genes that possibly induce tumor progression and drug resistance (42). According to this finding, we propose that the miRNA-320 family serves as potential therapeutic targets for the future management of HGSC.

This study has several limitations. First, the sample was relatively small since very few HGSC cases do not receive chemotherapy in routine clinical practice. In addition, determining miRNA expression profiles in CAFs could aid the diagnosis and treatment of HGSC. Second, in situ hybridization may be required to identify the expression of specific miRNAs in target cells. However, we did not perform in situ hybridization because it is a very difficult procedure in our laboratory. Alternatively, we examined whether stromal tissue is composed of pure CAFs in examined isolated stromal tissue, as a result, the histological finding of isolated stromal tissue was considered to be a CAF.

In conclusion, we examined miRNA expression by high-throughput genome-wide screening in isolated cancer glands and the surrounding stromal tissue separately. We found that downregulation of miRNA-214-3p in isolated cancer glands and downregulation of miRNA-320c in stromal tissue each correlated with a lack of recurrence (disease-free survival). This information can be valuable to increase the number of prognostic markers and treatment options for HGSC. Additional studies will be needed to verify the results seen for the present study.

\section{Acknowledgements}

The authors would like to thank Ms. E. Sugawara and Ms. C. Ishikawa (Department of Molecular Diagnostic
Pathology, School of Medicine, Iwate Medical University) for their technical assistance.

\section{Funding}

No funding was received.

\section{Availability of data and materials}

The datasets used and/or analyzed during the current study are available from the corresponding author on reasonable request.

\section{Authors' contributions}

CS constructed the figures and tables, and performed the statistical analyses. MO generated the figures and tables, and performed the statistical analyses. TN, HI and TB provided the clinical data, and examined the association between molecular findings and clinical features. HS assisted with the molecular techniques. TS contributed to the preparation of the manuscript, including all aspects of the data collection and analysis. All authors read and approved the final manuscript.

\section{Ethics approval and consent to participate}

All patients provided written informed consent, and the study was approved by the Iwate Medical University Institutional Review Board (approval no. MH2018-528).

\section{Patient consent for publication}

Not applicable.

\section{Competing interests}

The authors declare that they have no competing interests.

\section{References}

1. Reid BM, Permuth JB and Sellers TA: Epidemiology of ovarian cancer: A review. Cancer Biol Med 14: 9-32, 2017.

2. Lisio MA, Fu L, Goyeneche A, Gao ZH and Telleria C High-grade serous ovarian cancer: Basic sciences, clinical and therapeutic standpoints. Int J Mol Sci 20: 952, 2019.

3. Bray F, Ferlay J, Soerjomataram I, Siegel RL, Torre LA and Jemal A: Global cancer statistics 2018: GLOBOCAN estimates of incidence and mortality worldwide for 36 cancers in 185 countries. CA Cancer J Clin 68: 394-424, 2018.

4. Falzone L, Salomone S and Libra M: Evolution of cancer pharmacological treatments at the turn of the third millennium. Front Pharmacol 9: 1300, 2018.

5. Chandra A, Pius C, Nabeel M, Nair M, Vishwanatha JK, Ahmad S and Basha R: Ovarian cancer: Current status and strategies for improving therapeutic outcomes. Cancer Med 8: 7018-7031, 2019.

6. Mathieu KB, Bedi DG, Thrower SL, Qayyum A and Bast RC Jr: Screening for ovarian cancer: Imaging challenges and opportunities for improvement. Ultrasound Obstet Gynecol 51: 293-303, 2018.

7. Mills GB, Bast RC Jr and Srivastava S: Future for ovarian cancer screening: Novel markers from emerging technologies of transcriptional profiling and proteomics. J Natl Cancer Inst 93: 1437-1439, 2001.

8. Bandera CA, Ye B and Mok SC: New technologies for the identification of markers for early detection of ovarian cancer. Curr Opin Obstet Gynecol 15: 51-55, 2003. 
9. Zhang H, Liu T, Zhang Z, Payne SH, Zhang B, McDermott JE, Zhou JY, Petyuk VA, Chen L, Ray D, et al: Integrated proteogenomic characterization of human high-grade serous ovarian cancer. Cell 166: 755-765, 2016.

10. Cancer Genome Atlas Research Network: Integrated genomic analyses of ovarian carcinoma. Nature 474: 609-615, 2011.

11. Davidson B: Biomarkers of drug resistance in ovarian cancer-an update. Expert Rev Mol Diagn 19: 469-476, 2019.

12. Elias KM, Guo J and Bast RC Jr: Early detection of ovarian cancer. Hematol Oncol Clin North Am 32: 903-914, 2018.

13. Falzone L, Romano GL, Salemi R, Bucolo C, Tomasello B, Lupo G, Anfuso CD, Spandidos DA, Libra M and Candido S: Prognostic significance of deregulated microRNAs in uveal melanomas. Mol Med Rep 19: 2599-2610, 2019.

14. Staicu CE, Predescu DV, Rusu CM, Radu BM, Cretoiu D, Suciu N, Crețoiu SM and Voinea SC: Role of microRNAs as clinical cancer biomarkers for ovarian cancer: A short overview. Cells 9: 169, 2020

15. Yokoi A, Matsuzaki J, Yamamoto Y, Yoneoka Y, Takahashi K Shimizu H, Uehara T, Ishikawa M, Ikeda SI, Sonoda T, et al: Integrated extracellular microRNA profiling for ovarian cancer screening. Nat Commun 9: 4319, 2018.

16. Falzone L, Lupo G, La Rosa GRM, Crimi S, Anfuso CD, Salemi R, Rapisarda E, Libra M and Candido S: Identification of novel MicroRNAs and their diagnostic and prognostic significance in oral cancer. Cancers (Basel) 11: 610, 2019.

17. Cirri P and Chiarugi P: Cancer associated fibroblasts: The dark side of the coin. Am J Cancer Res 1: 482-497, 2011.

18. Cirri P and Chiarugi P: Cancer-associated-fibroblasts and tumour cells: A diabolic liaison driving cancer progression. Cancer Metastasis Rev 31: 195-208, 2012.

19. Dasari S, Fang Y and Mitra AK: Cancer associated fibroblasts Naughty neighbors that drive ovarian cancer progression. Cancers (Basel) 10: 406, 2018.

20. Sun W and Fu S: Role of cancer-associated fibroblasts in tumor structure, composition and the microenvironment in ovarian cancer. Oncol Lett 18: 2173-2178, 2019.

21. Japanese Society of Obstetrics and Gynecology, the Japanese Society of Pathology: The General Rules for Clinical and Pathological Management of Ovarian Tumors Part 1: Histological Classification and Color Atlas of Ovarian Tumors. 2nd edition. Kanehara, pp1-41, 2009.

22. Prat $\mathbf{J}$ and FIGO Committee on Gynecologic Oncology: Staging classification for cancer of the ovary, fallopian tube, and peritoneum. Int J Gynaecol Obstet 124: 1-5, 2014.

23. Sato A, Fujita Y, Otsuka K, Sasaki A, Suzuki H, Matsumoto T and Sugai T: Differential expression of microRNAs in colorectal cancer: Different patterns between isolated cancer gland and stromal cells. Pathol Int 70: 21-30, 2019.

24. Habano W, Sugai T, Nakamura S and Yoshida T: A novel method for gene analysis of colorectal carcinomas using a crypt isolation technique. Lab Invest 74: 933-940, 1996.

25. Sugai T, Habano W, Nakamura SI, Uesugi N, Sasou S and Itoh C: A unique method for mutation analysis of tumor suppressor genes in colorectal carcinomas using a crypt isolation technique. Arch Pathol Lab Med 124: 382-386, 2000.

26. Miles GD, Seiler M, Rodriguez L, Rajagopal G and Bhanot G: Identifying microRNA/mRNA dysregulations in ovarian cancer. BMC Res Notes 5: 164, 2012.

27. Shiomi E, Sugai T, Ishida K, Osakabe M, Tsuyukubo T, Kato Y, Takata R and Obara W: Analysis of expression patterns of microRNAs that are closely associated with renal carcinogenesis. Front Oncol 9: 431, 2019.
28. Kohlhapp FJ, Mitra AK, Lengyel E and Peter ME: MicroRNAs as mediators and communicators between cancer cells and the tumor microenvironment. Oncogene 34: 5857-5868, 2015.

29. Kossaï M, Leary A, Scoazec JY and Genestie C: Ovarian cancer: A heterogeneous disease. Pathobiology 85: 41-49, 2018

30. Comerford SA, Clouthier DE, Hinnant EA and Hammer RE: Induction of hepatocyte proliferation and death by modulation of T-Antigen expression. Oncogene 22: 2515-2530, 2003.

31. Li Y, Li Y, Chen Y, Xie Q, Dong N, Gao Y, Deng H, Lu C and Wang S: MicroRNA-214-3p inhibits proliferation and cell cycle progression by targeting MELK in hepatocellular carcinoma and correlates cancer prognosis. Cancer Cell Int 17: 102, 2017.

32. Phatak P, Byrnes KA, Mansour D, Liu L, Cao S, Li R, Rao JN, Turner DJ, Wang JY and Donahue JM: Overexpression of miR-214-3p in esophageal squamous cancer cells enhances sensitivity to cisplatin by targeting survivin directly and indirectly through CUG-BP1. Oncogene 35: 2087-2097, 2016.

33. Du T, Qu Y, Li J, Li H, Su L, Zhou Q, Yan M, Li C, Zhu Z and Liu B: Maternal embryonic leucine zipper kinase enhances gastric cancer progression via the FAK/Paxillin pathway. Mol Cancer 13: 100, 2014.

34. Yang Y, Li Z, Yuan H, Ji W, Wang K, Lu T, Yu Y, Zeng Q, Li F, $\mathrm{Xia} W$ and Lu S: Reciprocal regulatory mechanism between miR-214-3p and FGFR1 in FGFR1-amplified lung cancer. Oncogenesis 8: 50, 2019.

35. Falzone L, Candido S, Salemi R, Basile MS, Scalisi A, McCubrey JA, Torino F, Signorelli SS, Montella M and Libra M: Computational identification of microRNAs associated to both epithelial to mesenchymal transition and NGAL/MMP-9 pathways in bladder cancer. Oncotarget 7: 72758-72766, 2016.

36. Wang $\mathrm{J}, \mathrm{Xu} \mathrm{Y}$, Wang $\mathrm{J}$ and Ying $\mathrm{H}$ : Circulating miR-214-3p predicts nasopharyngeal carcinoma recurrence or metastasis. Clin Chim Acta 503: 54-60, 2020.

37. Vishnubalaji R, Hamam R, Yue S, Al-Obeed O, Kassem M, Liu FF, Aldahmash A and Alajez NM: MicroRNA 320 suppresses colorectal cancer by targeting SOX4, FOXM1, and FOXQ1. Oncotarget 7: 35789-35802, 2016.

38. Sun JY, Huang Y, Li JP, Zhang X, Wang L, Meng YL, Yan B, Bian YQ, Zhao J, et al: MicroRNA-320a suppresses human colon cancer cell proliferation by directly targeting $\beta$-catenin. Biochem Biophys Res Commun 420: 787-792, 2012.

39. Zhao H, Dong T, Zhou H, Wang L, Huang A, Feng B, Quan Y, Jin R, Zhang W, Sun J, et al: MiR-320a suppresses colorectal cancer progression by targeting Racl. Carcinogenesis 35: 886-895, 2014

40. Zhang H, Li W, Nan F, Ren F, Wang H, Xu Y and Zhang F: MicroRNA expression profile of colon cancer stem-like cells in HT29 adenocarcinoma cell line. Biochem Biophys Res Commun 404: 273-278, 2011.

41. Chen L, Yan HX, Yang W, Hu L, Yu LX, Liu Q, Li L, Huang DD, Ding J, Shen F, et al: The role of microRNA expression pattern in human intrahepatic cholangiocarcinoma. J Hepatol 50: 358-369, 2009.

42. Hsieh IS, Chang KC, Tsai YT, Ke JY, Lu PJ, Lee KH, Yeh SD, Hong TM and Chen YL: MicroRNA-320 suppresses the stem cell-like characteristics of prostate cancer cells by downregulating the Wnt/beta-catenin signaling pathway. Carcinogenesis 34 : $530-538,2013$.

This work is licensed under a Creative Commons Attribution-NonCommercial-NoDerivatives 4.0 International (CC BY-NC-ND 4.0) License. 